\title{
Absolute neutrino mass and the Dirac/Majorana distinction from the weak interaction of aggregate matter
}

\author{
Alejandro Segarra $\odot$ and José Bernabéu \\ Department of Theoretical Physics, University of Valencia and IFIC, University Valencia-CSIC,
} Burjassot, Valencia E-46100, Spain

(Received 18 January 2020; revised manuscript received 9 March 2020; accepted 22 April 2020; published 14 May 2020)

\begin{abstract}
The $2 \nu$-mediated force has a range of microns, well beyond the atomic scale. The effective potential is built from the $t$-channel absorptive part of the scattering amplitude and depends on neutrino properties on shell. We demonstrate that neutral aggregate matter has a weak charge and calculate the matrix of six coherent charges for its interaction with definite-mass neutrinos. Near the range of the potential the neutrino pair is nonrelativistic, leading to observable absolute mass and Dirac/Majorana distinction via different $r$-dependence and violation of the weak equivalence principle.
\end{abstract}

DOI: 10.1103/PhysRevD.101.093004

\section{INTRODUCTION}

The experimental evidence of neutrino oscillations is one of the most important discoveries in particle physics. The first evidence of model-independent neutrino oscillations was obtained in 1998 by the Super-Kamiokande atmospheric neutrino experiment [1], in 2002 by the SNO solar neutrino experiment [2], and later with reactor [3] and accelerator [4] neutrinos. The existence of neutrino oscillations implies that neutrinos are massive particles and that the three flavor neutrinos $\nu_{e}, \nu_{\mu}, \nu_{\tau}$ are mixtures of the neutrinos with definite masses $\nu_{i}$ (with $i=1,2,3$ ). The phenomenon of neutrino oscillations is being studied in a variety of experiments which fully confirm this fundamentally quantum phenomenon in different disappearance and appearance channels. The mixing matrix $U_{\text {PMNS }}[5,6]$ contains three mixing angles, already known, and one $C P$-violating phase for flavor oscillations. This quantum interference phenomenon measures the phase differences due to the squared mass splittings $\Delta m_{21}^{2}$ and $\left|\Delta m_{31}^{2}\right|$, but the absolute mass scale is inaccessible. The answer to this last open question is being sought after by the KATRIN experiment [7] in tritium beta decay, with an expected final sensitivity around $0.2 \mathrm{eV}$.

Knowing that neutrinos are massive, the most fundamental problem is the determination of the nature of neutrinos with definite mass: are they either four-component Dirac particles with a conserved global lepton number $L$, distinguishing neutrinos from antineutrinos, or two-component

Published by the American Physical Society under the terms of the Creative Commons Attribution 4.0 International license. Further distribution of this work must maintain attribution to the author(s) and the published article's title, journal citation, and DOI. Funded by SCOAP ${ }^{3}$. truly neutral (no electric charge and no global lepton number) self-conjugate Majorana particles [8]? For Dirac neutrinos, like quarks and charged leptons, their masses can be generated in the Standard Model of particle physics by spontaneous breaking of the gauge symmetry with the doublet Higgs scalar, if there were additional right-handed sterile neutrinos. But the Yukawa couplings would then be unnaturally small compared to those of all other fermions. One would also have to explain the origin of the global lepton number avoiding a Majorana mass for these sterile neutrinos. A Majorana $\Delta L=2$ mass term, with the active left-handed neutrinos only, leads to definite-mass neutrinos with no definite lepton charge. However, there is no way in the Standard Model to generate this Majorana mass, so the important conclusion in fundamental physics arises: Majorana neutrinos would be an irrefutable proof of physics beyond the Standard Model. Due to the Majorana condition of neutrinos with definite mass being their own antiparticles, Majorana neutrinos have two additional $C P$-violating phases [9-11] beyond the Dirac case.

Neutrino flavor oscillation experiments cannot determine the fundamental nature of massive neutrinos. In order to probe whether neutrinos are Dirac or Majorana particles, the known way has been the search of processes violating the global lepton number $L$. The difficulty encountered in these studies is well illustrated by the so-called confusion theorem $[12,13]$, stating that in the limit of zero mass there is no difference between the left component of Dirac neutrinos and Majorana neutrinos, both of them coinciding with Weyl neutrinos. As all known neutrino sources produce highly relativistic neutrinos (except for the present cosmic neutrino background in the universe), the $\Delta L=2$ observables are highly suppressed. Up to now, there is a consensus that the highest sensitivity to small Majorana neutrino masses can be reached in experiments on the search of the $L$-violating neutrinoless double- $\beta$ decay 
process $(0 \nu \beta \beta)$. Dozens of experiments around the world are seeking out a positive signal, and the most sensitive limits are obtained by GERDA-II [14] in ${ }^{76} \mathrm{Ge}$, CUORE [15] in ${ }^{130} \mathrm{Te}$ and KAMLAND-Zen [16] in ${ }^{136} \mathrm{Xe}$. An alternative to $0 \nu \beta \beta$ is provided by the mechanism of neutrinoless double electron capture $(0 \nu \mathrm{ECEC})$ [17], which actually corresponds to a virtual mixing between a nominally stable parent $Z$ atom and a daughter $(Z-2)^{*}$ atom with two electron holes. The experimental process is the subsequent x-ray emission and it becomes resonantly enhanced when the two mixed atomic states are nearly degenerate. The results of Ref. [17] were improved on the theoretical treatment and the promising nuclides in Refs. [18,19]. The process can be stimulated [20] in XLaser facilities. The $2 \nu$ ECEC decay, allowed in the Standard Model, has recently been observed for the first time by the XENON collaboration [21] in ${ }^{124} \mathrm{Xe}$. This last process, contrary to the case of $2 \nu \beta \beta$ for searches of $0 \nu \beta \beta$, is not an irreducible background for $0 \nu$ ECEC when the resonance condition is satisfied.

In this paper we present and develop a novel idea for this dilemma, following a different path to the search of $\Delta L=2$ processes. It is based on having a pair of virtual nonrelativistic neutrinos of definite mass, whose quantum distinguishability is different for Dirac and Majorana nature due to the lepton charge. Such a physical situation is apparent in the long-range force mediated by two neutrinos at distances near its range. There is a conjunction of facts that cooperate in the achievement of this goal:

(1) The Compton wavelength of massive neutrinos is of order 1 micron. Although the absolute scale of neutrino masses is still unknown, the present upper limit and the known $\left|\Delta m_{31}^{2}\right|$ and $\Delta m_{21}^{2}$ values-see, for example, Ref. [22]—tell us that $m_{\nu} \sim 0.1 \mathrm{eV}$ can be taken as a reference. Such a range for the twoneutrino-mediated force is well above the atomic scale, so the force will be operative for atoms and aggregate matter if they have a weak charge, being neutral in electric charge.

(2) Indeed a coherent weak charge [23-25] is built from neutral-current interactions of neutrinos with electrons, protons and neutrons, and the charged-current interaction of electron neutrinos with electrons. These different weak charges for $\nu_{e}$ and $\nu_{\mu, \tau}$ are proportional to the number operator and thus they violate the weak equivalence principle (WEP).

(3) For neutral-current interactions, flavor mixing is unoperative and the intermediate neutrino propagation with definite mass directly appears. For the charged-current interaction, the mixing $U_{e i}$ of electron neutrinos to all neutrinos of definite mass will be needed. This ingredient is also well known [22] from neutrino oscillation experiments.

(4) The dispersion theory of long-range forces leads to the effective potential in terms of the absorptive part of the amplitude at low $t$, i.e., the energy of the neutrino pair in the $t$ channel. Hence the physics involved, by unitarity, is that of a pair of low energy neutrinos with definite mass. One then expects a Hermitian matrix with six different weak charges. The only unknown is the lightest neutrino mass.

(5) For Dirac neutrinos with definite lepton charge, the interaction vertex is the chiral charge distinguishing neutrinos from antineutrinos. For Majorana neutrinos with no conserved charge, the interaction vertex is twice the axial charge and so, contrary to the Dirac case, the pair is in $\mathrm{P}$ wave. The absorptive parts for Dirac and Majorana neutrinos will differ in the massdependent terms leading to different $r$-dependent potentials near their range.

(6) Formidable precision tests of WEP and the $r$ dependence of forces between matter aggregates are being pursued in recent years. They reached the centimeter to micron scale of distances from different approaches like torsion balance [26,27], optical levitation [28] and atom interferometry $[29,30]$. When interpreted in terms of a new "fifth force" Yukawa interaction, the present upper limit to its coupling relative to gravity goes [27] from 1 to $10^{6}$ for a corresponding range from 40 to 1 micron.

Following these guiding concepts, we have developed [31] the theory of the long-range force mediated by two neutrinos including for the first time all ingredients of neutrino physics relevant to the region of distances near the range of the interaction, with masses, mixing and the Dirac/ Majorana distinction.

\section{II. $2 \nu$-EXCHANGE POTENTIAL}

The dispersion theory of long-range forces was developed in Ref. [32] for the two-photon mediation between neutral objects, reproducing in a model-independent way the Casimir-Polder potential [33]. The method was later applied to the case of charged-neutral objects [34] and extended for a low-momentum-transfer theorem in leptonhadron scattering [35]. The two-neutrino mediation was given in 1968 [36] for charged-current interactions of two electrons and, in the dispersion approach, it was later extended [23-25] considering neutral current interactions as well. With the advent of neutrino masses and mixings, the long range $r^{-5}$ potential will be modified at distances near its range. For Dirac neutrinos, these effects have been calculated [37] for electrons and nucleons using oldfashioned perturbation theory. These particle-particle cases are consistent with our inputs for the constituents of ordinary matter. In this work we present the results for the effective potential between aggregate matter obtained in the dispersion approach, with appropriate treatment of the different mass terms corresponding to either Dirac or Majorana neutrinos. Thus we open an alternative path to 
the known search of forbidden $\Delta L=2$ processes. We demonstrate that, at distances $1-10$ microns, the potential is extremely sensitive to the mass of the lightest neutrino varying between 0 and $0.1 \mathrm{eV}$ and to the Dirac/Majorana distinction.

The Feynman diagram describing the two-neutrinomediated interaction between two objects $\mathrm{A}$ and $\mathrm{B}$ is depicted in Fig. 1. In the dispersion approach the potential is given by the integral transform of the $t$-channel absorptive part of the scattering amplitude $A B \rightarrow A B$. The longest range contribution comes, for three neutrino species, from the six amplitudes $M_{i j}(t)$ and thresholds $t_{i j}=\left(m_{i}+m_{j}\right)^{2}$, such that

$$
V(r)=\frac{-1}{4 \pi^{2} r} \sum_{i j} \int_{t_{i j}}^{\infty} \mathrm{d} t e^{-\sqrt{t} r} \operatorname{Im} \mathcal{M}_{i j}(t) .
$$

We already appreciate in Eq. (1) the complementarity, in the quantum-mechanical sense, between long distances $r$ and low $t$ behavior of the absorptive parts. Using unitarity, they are given by the $t$-channel crossing for $A \bar{A} \rightarrow B \bar{B}[38]$ :

$$
\begin{aligned}
\operatorname{Im} \mathcal{M}_{i j}^{A \bar{A} \rightarrow B \bar{B}}= & \frac{1}{2} \int \frac{\mathrm{d}^{4} k_{1}}{(2 \pi)^{3}} \delta\left(k_{1}^{2}-m_{i}^{2}\right) \frac{\mathrm{d}^{4} k_{2}}{(2 \pi)^{3}} \delta\left(k_{2}^{2}-m_{j}^{2}\right) \\
& \times(2 \pi)^{4} \delta^{(4)}\left(k_{1}+k_{2}-p_{i}\right) \mathcal{M}_{B \bar{B} \rightarrow \nu_{i} \bar{\nu}_{j}}^{*} \mathcal{M}_{A \bar{A} \rightarrow \nu_{i} \bar{\nu}_{j}} .
\end{aligned}
$$

As seen, the intermediate states are pairs $(i, j)$ of definite-mass neutrinos connected to their interaction vertices with matter. For neutral-current interactions there are diagonal $i=j$ terms only, interacting with electrons, protons and neutrons. For charged-current interactions there are nondiagonal terms $i \neq j$ too for electron neutrinos $(\alpha=e)$ interacting with electrons by means of the mixing $U_{e i} U_{e j}^{*}$.

The low-energy interaction of definite-mass neutrinos with the matter constituents is given by the effective Lagrangian in the Standard Model

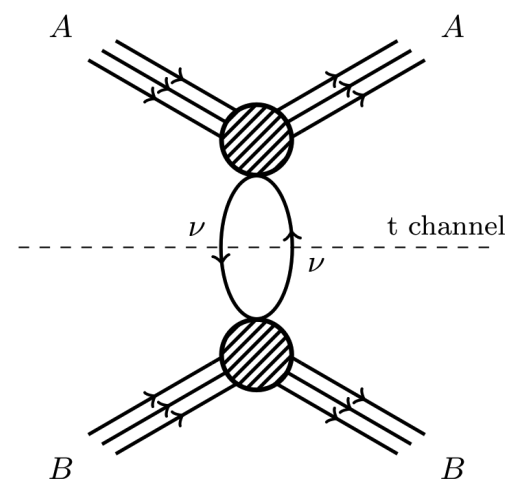

FIG. 1. Leading-order Feynman diagram for the neutrino-pair mediated $t$-channel $A B \rightarrow A B$ scattering.
$\mathcal{L}_{\text {eff }}=-\frac{G_{F}}{2 \sqrt{2}}\left[\bar{\nu}_{j} \gamma^{\mu}\left(1-\gamma_{5}\right) \nu_{i}\right]\left[\bar{\psi} \gamma_{\mu}\left(g_{V_{i j}}^{\psi}-g_{A_{i j}}^{\psi} \gamma_{5}\right) \psi\right]$,

with $\psi=e, p, n$ describing the fermion fields of the constituents. For aggregate matter, we are interested in the current component proportional to the number operator, the $\mu=0$ component of the vector current. The other components yield relativistic or spin-dependent noncoherent interactions. Thus the only relevant couplings are

$$
\begin{aligned}
& g_{V_{i j}}^{e}=2 U_{e i} U_{e j}^{*}-\left(1-4 \sin ^{2} \theta_{W}\right) \delta_{i j}, \\
& g_{V_{i j}}^{p}=\left(1-4 \sin ^{2} \theta_{W}\right) \delta_{i j}, \\
& g_{V_{i j}}^{n}=-\delta_{i j},
\end{aligned}
$$

and the coherent global weak charges are given by the six independent elements of the Hermitian matrix,

$$
Q_{W}^{i j}(Z, N)=Z\left(g_{V_{i j}}^{e}+g_{V_{i j}}^{p}\right)+N g_{V_{i j}}^{n},
$$

where both the atomic number $Z$ and the neutron number $N$ have to be multiplied by the number of neutral atoms in the A (or B) object. As gravity, we have here a coherent interaction for aggregate matter, however not proportional to the mass of the object, thus leading to a violation of WEP.

In the calculation of the absorptive part in Eq. (2) there are both dynamical and kinematical mass effects. For Dirac neutrinos, the right-handed components of the states with definite mass are sterile, so their interaction continues to be the V-A chiral charge. Majorana neutrino states of definite mass, on the other hand, have their two chiralities of lefthanded neutrino and its conjugate as active interacting components. This is the case because of the $\Delta L=2$ Majorana mass term in the Langrangian, connecting the left-handed field and its conjugate. As a consequence, the interaction vertex of this state is twice the axial current and, when contracted with the coherent weak charge of matter, the intervening contribution is the parity-odd axial charge. This fundamental difference in the dynamics of Majorana neutrinos, with respect to Dirac neutrinos, is affecting the behavior of the absorptive part at low values of $t$. The results for either Dirac or Majorana absorptive parts are

$$
\begin{aligned}
\operatorname{Im} \mathcal{M}_{i j}= & -\frac{G_{F}^{2}}{48 \pi} t Q_{W, A}^{i j} Q_{W, B}^{i j *} \sqrt{1-\frac{4 \overline{m_{i j}^{2}}}{t}+\left[\frac{\Delta m_{i j}^{2}}{t}\right]^{2}} \\
& \times\left[1-\frac{1}{t}\left\{\frac{\overline{m_{i j}^{2}}}{m_{i j}^{2}}+3 m_{i} m_{j}\right\}-\frac{1}{2}\left[\frac{\Delta m_{i j}^{2}}{t}\right]^{2}\right],
\end{aligned}
$$

where $\overline{m_{i j}^{2}} \equiv \frac{1}{2}\left(m_{i}^{2}+m_{j}^{2}\right), \Delta m_{i j}^{2} \equiv m_{i}^{2}-m_{j}^{2}$, and the upper/ lower dynamical terms in the bracket correspond to Dirac/ 
Majorana neutrinos, whereas mass effects in the first line are kinematical and thus blind to the neutrino nature.

Several relevant comments are in order: (i) the charges are now specific to the $(i, j)$ intermediate channels as $Q_{W}^{i j}=2 Z U_{e i} U_{e j}^{*}-N \delta_{i j}$ in Eq. (5); (ii) the complex mixings enter the absorptive part, although only as the $\left|U_{e i}\right|$ moduli even for $A \neq B$ matter aggregates, so that no $C P$ violation effects are accessible; (iii) Eq. (6) leads in the limit of vanishing masses to the correct linear $t$ dependence; (iv) the Dirac/Majorana distinction appears in the mass terms, and they reproduce the case of a single neutrino species [39] considered in the context of neutron stars; (v) for nonrelativistic neutrinos with momenta $k$ in the CM frame $t=t_{0}\left(1+k^{2} / m_{i} m_{j}\right)$, so the absorptive part of the $(i, j)$ channel is either S-wave proportional to $k$ for Dirac neutrinos or $\mathrm{P}$-wave proportional to $k^{3}$ for Majorana neutrinos. This different nonrelativistic behavior leads to the final distinction in the potential, providing the signal to determine the neutrino nature.

\section{A. The coherent weak flavor charge of neutral matter}

If the lightest neutrino is massless, its contribution will dominate the behavior of the potential at the longest distances with the known $r^{-5}$ dependence. At distances such that $m_{i} r \ll 1 \forall i$, the exchanged neutrinos are extremely relativistic, thus leading to a common absorptive part linear in $t$. In this last case, there is a global A-B coupling of coherent weak flavor charges $Q_{W}^{\alpha}$,

$$
\sum_{i j} Q_{W, A}^{i j}\left(Q_{W, B}^{i j}\right)^{*}=\sum_{\alpha} Q_{W, A}^{\alpha} Q_{W, B}^{\alpha},
$$

as a sum extended to all diagonal neutrino $\alpha$-flavors, with $Q_{W}^{e}=2 Z-N$ and $Q_{W}^{\mu, \tau}=-N$ for each single atom. These weak flavor charges are represented in Fig. 2 for the most

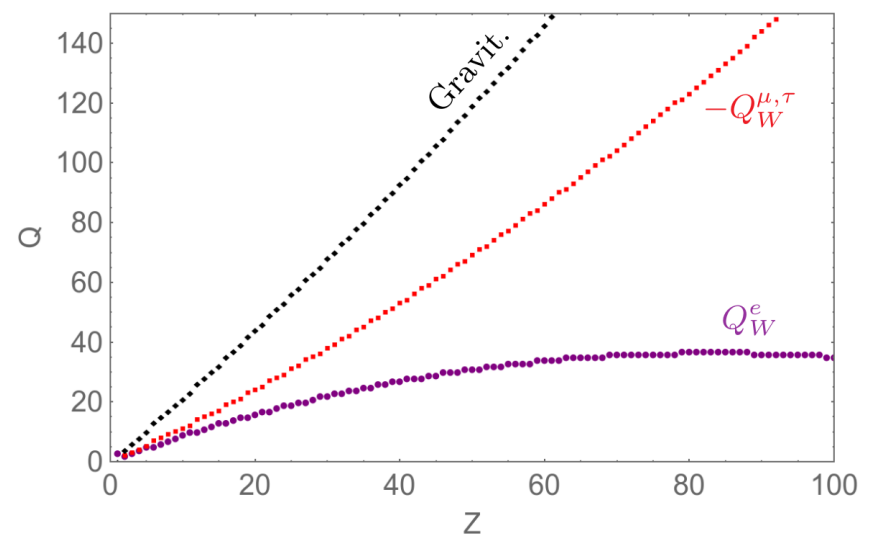

FIG. 2. $\quad e$ (purple filled circle), $\mu$ and $\tau$ (red filled square) weak flavor charges of the elements with $(Z, N)$ in the valley of stability, as well as their gravitational coupling, approximately proportional to $Z+N$ (black filled diamond). Beware a minus sign in the $\mu, \tau$ flavor charges. stable isotopes, following a semiempirical formula [40] relating $Z$ and $N$. The $Z$ dependencies of the weak flavor charges are compared with the $Z+N(Z)$ curve approximately giving the mass coupling for gravity. In this limit, using Eq. (7) for aggregate matter reproduces the known repulsive potential

$$
V\left(m_{i} r \ll 1\right)=\frac{G_{F}^{2}}{16 \pi^{3}} \frac{1}{r^{5}} \sum_{\alpha} Q_{W, A}^{\alpha} Q_{W, B}^{\alpha},
$$

describing the coherent interaction above the atomic scale.

\section{B. The $r$-dependence near the range}

Having identified the physics responsible of the Dirac/ Majorana neutrino nature distinction in our interaction potential between two objects of ordinary matter, we find it convenient to show explicit analytic results for the $r$ dependence near its range. Expanding Eq. (6) and inserting it in the integral transform (1), we obtain the contribution of each $(i, j)$ intermediate channel,

$$
\begin{aligned}
V\left(m_{i} r>1\right)= & \frac{G_{F}^{2}}{64 \pi^{5 / 2}} \sum_{i j} Q_{W, A}^{i j}\left(Q_{W, B}^{i j}\right)^{*} e^{-r \sqrt{t_{i j}}} \\
& \times \frac{\sqrt{t_{i j}}}{r}\left[\frac{2 \mu_{i j}}{r}\right]^{3 / 2}\left[\left\{\begin{array}{l}
1 \\
0
\end{array}\right\}+\left(3-\frac{4 \mu_{i j}}{\sqrt{t_{i j}}}\right) \frac{1}{2 \mu_{i j} r}\right],
\end{aligned}
$$

where $\mu_{i j}=\frac{m_{i} m_{j}}{m_{i}+m_{j}}$ is the reduced mass of the $\nu_{i} \nu_{j}$ pair, $\sqrt{t_{i j}}=m_{i}+m_{j}$ and the $1 / 0$ in the bracket correspond to Dirac/Majorana neutrinos. The intermediate masses depend on the assumed either normal or inverted neutrino mass ordering and on the absolute mass $m_{\min }$ of the lightest neutrino. In Table I we give the neutrino masses assuming $m_{\min }=0$ and $m_{\min }=0.1 \mathrm{eV}$.

TABLE I. Absolute neutrino masses (in eV) for the extreme $m_{\min }$ values, and both normal and inverted hierarchies.

\begin{tabular}{lcl}
\hline \hline & $\mathrm{NH}$ & \\
\hline$m_{3}$ & 0.0500 & 0.1118 \\
$m_{2}$ & 0.0087 & 0.1004 \\
$m_{1}$ & 0 & 0.1 \\
\hline \hline \multicolumn{3}{c}{$\mathrm{IH}$} \\
\hline$m_{2}$ & 0.0500 & 0.1118 \\
$m_{1}$ & 0.0492 & 0.1115 \\
$m_{3}$ & 0 & 0.1 \\
\hline \hline
\end{tabular}




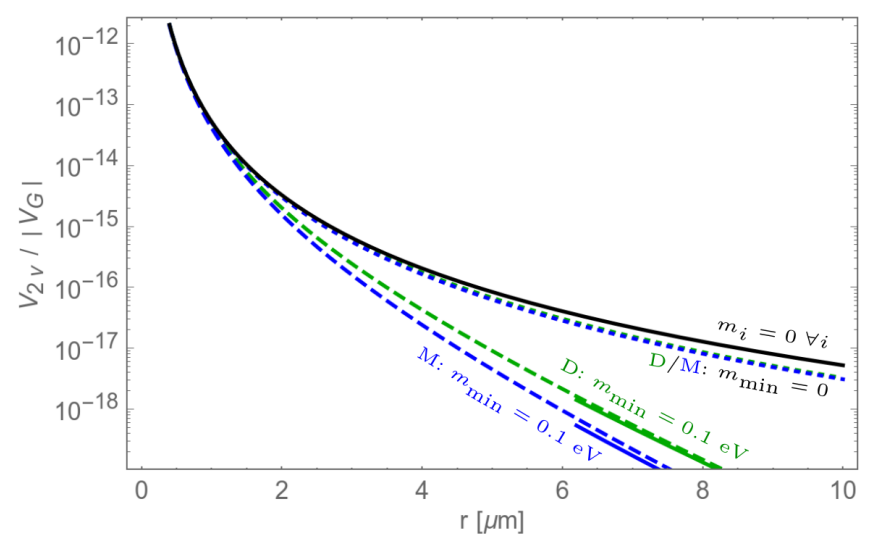

FIG. 3. $2 \nu$-mediated long-range potential between two atoms of ${ }^{56} \mathrm{Fe}$, relative to their gravitational potential. Numerical results for $m_{\min }=0$ (superimposed Dirac/Majorana dotted lines) and $m_{\min }=0.1 \mathrm{eV}$ (dashed Dirac and Majorana lines), together with analytic limits (solid lines) in Eqs. (8) and (9).

\section{RESULTS: SENSITIVITY TO ABSOLUTE $\nu$-MASS AND DIRAC VS MAJORANA}

All the ingredients are now ready to compute at all distances the two-neutrino-mediated potential $V(r)$, always repulsive for aggregate matter, with the analytic limits given in Eqs. (8) and (9). We compare its $r$ dependence with the attractive gravitational potential. The six coherent weak charges are matter dependent and their $Z$ behavior is different from the mass, opening the door to experimental studies based on the violation of WEP. The six $r$ dependencies are affected by the sought properties of absolute neutrino mass and the Dirac/ Majorana nature. Each $(i, j)$ channel has a different branching point $t_{i j}=\left(m_{i}+m_{j}\right)^{2}$, so the integral transforms of their absorptive parts have to be computed separately. It is important to study whether the observable convoluted potential, built from the six intermediate neutrino-pair exchanges, still keeps near its range the precious information on the neutrino properties.

We perform numerically the integrals for the atom-atom interaction with the most stable nucleus, ${ }^{56} \mathrm{Fe}$, and show the resulting Dirac and Majorana $2 \nu$-exchange potentials between 1 and 10 microns in Fig. 3. This result demonstrates the existing major difference in that region of distances due to the value of $m_{\min }$ and the distinct $r$ dependence for Dirac and Majorana neutrino natures. Indeed, Eq. (9) explains the higher suppression of larger neutrino mass contributions, as well as the S-wave Dirac potential being larger than the P-wave Majorana one. On the other hand, the two possible neutrino hierarchies, corresponding to the masses in Table I, do not give an appreciable difference.

\section{CONCLUSIONS}

To summarize, a novel concept to search for the elusive neutrino properties of absolute mass and Dirac/Majorana nature has been presented. It is based on the existence of a coherent weak charge for neutral aggregate matter, with anticipated deviations of the weak equivalence principle, as well as the $r$ dependence of the two-neutrino-mediated potential at distances near its range. A novel methodology consisting in the (virtual) exploration of nonrelativistic neutrinos with a different behavior depending on their massive nature is thus in place, complementary to the known approaches. The results in Fig. 3 for atom-atom interactions can be extended to sources of aggregate matter coherently enhancing both weak and gravitational potentials while keeping the appreciable sensitivity to these fundamental neutrino properties. It remains to be seen whether a terrestrial experiment could measure this weak observable by means of devices able to cancel the gravitational effects of the Earth, the major obstacle to overcome. Current studies in this direction, looking for a conceptual design, are being pursued.

\section{ACKNOWLEDGMENTS}

We would like to thank F. Botella, A. Magnon, S. Palomares, D. O. Sabulsky, F. Sánchez, and A. Santamaría for enlightening discussions. This research has been supported by the FEDER/MCIyU-AEI Grant No. FPA2017-84543-P, Severo Ochoa Excellence Centre Project No. SEV 2014-0398, and Generalitat Valenciana Projects No. GV PROMETEO 2017-033 and No. GV PROMETEO 2019-113. One of us (A. S.) acknowledges the Spanish Ministry of Education (MECD) support through the FPU14/04678 grant.
[1] Y. Fukuda et al. (Super-Kamiokande Collaboration), Evidence for Oscillation of Atmospheric Neutrinos, Phys. Rev. Lett. 81, 1562 (1998).

[2] Q. R. Ahmad et al. (SNO Collaboration), Direct Evidence for Neutrino Flavor Transformation from Neutral Current
Interactions in the Sudbury Neutrino Observatory, Phys. Rev. Lett. 89, 011301 (2002).

[3] T. Araki et al. (KamLAND Collaboration), Measurement of Neutrino Oscillation with KamLAND: Evidence of Spectral Distortion, Phys. Rev. Lett. 94, 081801 (2005). 
[4] M. H. Ahn et al. (K2K Collaboration), Measurement of neutrino oscillation by the K2K experiment, Phys. Rev. D 74, 072003 (2006).

[5] B. Pontecorvo, Mesonium and anti-mesonium, $\mathrm{Zh}$. Eksp. Teor. Fiz. 33, 549 (1957) [Sov. Phys. JETP 6, 429 (1957)].

[6] Z. Maki, M. Nakagawa, and S. Sakata, Remarks on the unified model of elementary particles, Prog. Theor. Phys. 28, 870 (1962).

[7] M. Aker et al. (KATRIN Collaboration), An Improved Upper Limit on the Neutrino Mass from a Direct Kinematic Method by KATRIN, Phys. Rev. Lett. 123, 221802 (2019).

[8] E. Majorana, Teoria simmetrica dell'elettrone e del positrone, Nuovo Cimento 14, 171 (1937).

[9] S. M. Bilenky, J. Hosek, and S. T. Petcov, On oscillations of neutrinos with Dirac and Majorana masses, Phys. Lett. 94B, 495 (1980).

[10] M. Doi, T. Kotani, H. Nishiura, K. Okuda, and E. Takasugi, $C P$ violation in Majorana neutrinos, Phys. Lett. 102B, 323 (1981).

[11] J. Bernabeu and P. Pascual, $C P$ properties of the leptonic sector for Majorana neutrinos, Nucl. Phys. B228, 21 (1983).

[12] K. M. Case, Reformulation of the Majorana theory of the neutrino, Phys. Rev. 107, 307 (1957).

[13] C. Ryan and S. Okubo, On the equivalence of the Majorana and two-component theories of the neutrino, Nuovo Cimento Suppl. 2, 234 (1964).

[14] M. Agostini et al. (GERDA Collaboration), Improved Limit on Neutrinoless Double- $\beta$ Decay of ${ }^{76} \mathrm{Ge}$ from GERDA Phase II, Phys. Rev. Lett. 120, 132503 (2018).

[15] D. Q. Adams et al. (CUORE Collaboration), Improved limit on neutrinoless double-beta decay in ${ }^{130} \mathrm{Te}$ with CUORE, arXiv:1912.10966.

[16] A. Gando et al. (KamLAND-Zen Collaborations), Search for Majorana Neutrinos Near the Inverted Mass Hierarchy Region with KamLAND-Zen, Phys. Rev. Lett. 117, 082503 (2016); 117, 109903 (2016).

[17] J. Bernabeu, A. De Rujula, and C. Jarlskog, Neutrinoless double electron capture as a tool to measure the $\nu_{e}$ mass, Nucl. Phys. B223, 15 (1983).

[18] M. Krivoruchenko, F. Simkovic, D. Frekers, and A. Faessler, Resonance enhancement of neutrinoless double electron capture, Nucl. Phys. A859, 140 (2011).

[19] S. Eliseev et al., Resonant Enhancement of Neutrinoless Double-Electron Capture in Gd-152, Phys. Rev. Lett. 106, 052504 (2011).

[20] J. Bernabéu and A. Segarra, Stimulated transitions in resonant atom Majorana mixing, J. High Energy Phys. 02 (2018) 017.

[21] E. Aprile et al. (XENON Collaboration), Observation of two-neutrino double electron capture in ${ }^{124} \mathrm{Xe}$ with XENON1T, Nature (London) 568, 532 (2019).

[22] P. F. de Salas, D. V. Forero, C. A. Ternes, M. Tortola, and J. W. F. Valle, Status of neutrino oscillations 2018: $3 \sigma$ hint for normal mass ordering and improved $C P$ sensitivity, Phys. Lett. B 782, 633 (2018).

[23] G. Feinberg, J. Sucher, and C. K. Au, The dispersion theory of dispersion forces, Phys. Rep. 180, 83 (1989).

[24] J. Bernabeu, Neutrino properties, Nucl. Phys. B, Proc. Suppl. 28, 500 (1992).

[25] S. D. H. Hsu and P. Sikivie, Long range forces from two neutrino exchange revisited, Phys. Rev. D 49, 4951 (1994).

[26] D. J. Kapner, T. S. Cook, E. G. Adelberger, J. H. Gundlach, B. R. Heckel, C. D. Hoyle, and H. E. Swanson, Tests of the Gravitational Inverse-Square Law Below the Dark-Energy Length Scale, Phys. Rev. Lett. 98, 021101 (2007).

[27] J. Lee, E. Adelberger, T. Cook, S. Fleischer, and B. Heckel, New Test of the Gravitational $1 / r^{2}$ Law at Separations Down to $52 \mu \mathrm{m}$, Phys. Rev. Lett. 124, 101101 (2020).

[28] A. D. Rider, D. C. Moore, C. P. Blakemore, M. Louis, M. Lu, and G. Gratta, Search for Screened Interactions Associated with Dark Energy Below the $100 \mu \mathrm{m}$ Length Scale, Phys. Rev. Lett. 117, 101101 (2016).

[29] M. Jaffe, P. Haslinger, V. Xu, P. Hamilton, A. Upadhye, B. Elder, J. Khoury, and H. Müller, Testing sub-gravitational forces on atoms from a miniature, in-vacuum source mass, Nat. Phys. 13, 938 (2017).

[30] D. O. Sabulsky, I. Dutta, E. A. Hinds, B. Elder, C. Burrage, and E. J. Copeland, Experiment to Detect Dark Energy Forces Using Atom Interferometry, Phys. Rev. Lett. 123, 061102 (2019).

[31] A. Segarra, Breaking of discrete symmetries and global lepton number in neutrino physics, Ph.D. thesis, University of Valencia, 2019.

[32] G. Feinberg and J. Sucher, General theory of the van der Waals interaction: A model-independent approach, Phys. Rev. A 2, 2395 (1970).

[33] H. B. G. Casimir and D. Polder, The influence of retardation on the London-van der Waals forces, Phys. Rev. 73, 360 (1948).

[34] J. Bernabeu and R. Tarrach, Long range potentials and the electromagnetic polarizabilities, Ann. Phys. (N.Y.) 102, 323 (1976).

[35] J. A. Penarrocha and J. Bernabeu, Low momentum transfer theorem in lepton hadron scattering, Ann. Phys. (N.Y.) 135, 321 (1981).

[36] G. Feinberg and J. Sucher, Long-range forces from neutrino-pair exchange, Phys. Rev. 166, 1638 (1968).

[37] Q. Le Thien and D.E. Krause, Spin-independent twoneutrino exchange potential with mixing and $C P$-violation, Phys. Rev. D 99, 116006 (2019).

[38] A. D. Martin and T.D. Spearman, Elementary-Particle Theory (North-Holland, Amsterdam, 1970).

[39] J. A. Grifols, E. Masso, and R. Toldra, Majorana neutrinos and long range forces, Phys. Lett. B 389, 563 (1996).

[40] K. S. Krane, Introductory Nuclear Physics (John Wiley \& Sons, New York, 1987). 